Pacific Journal of Mathematics

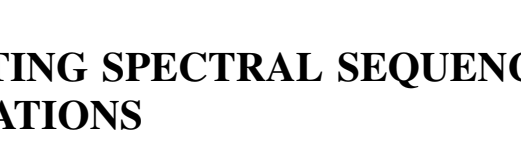




\title{
FILTERED SPACES ADMITTING SPECTRAL SEQUENCE OPERATIONS
}

\author{
LeWIS ShILANE
}

Introduction. Throughout this paper we will work in the category of compactly generated Hausdorff spaces. All cohomology will be singular cohomology with $Z_{2}$ coefficients, although similar results may be derived when 2 is replaced by an odd prime.

We will call a filtration $X_{0} \subseteq X_{1} \subseteq X_{2} \subseteq \cdots \subseteq X$ of a space $X$ convergent if $X=\cup X_{n}$ and every compact subset of $X$ is contained in some $X_{n}$. In this case the cohomology spectral sequence of $X$ converges. A subspace $A$ of $X$ has the induced filtration $A_{k} \equiv A \cap X_{k}$. A map $f: X \rightarrow Y$ will be called filtered if for each $n, f\left(X_{n}\right) \subseteq Y_{n}$. The product of two filtered spaces $X$ and $Y$ is given the "tensor product" filtration $(X \times Y)_{n} \equiv \cup_{i+\jmath=n} X_{\imath} \times Y_{J} . S^{\infty}$ will represent the infinite sphere with regular $C W$ reticulation consisting of two cells in each dimension, with the skeletal filtration, and with the antipodal $Z_{2}$ action.

We will define a category of convergent filtered spaces and prove that each object $Q$ has the following properties. First, the diagonal map $Q \stackrel{d_{O}}{\rightarrow} Q \times Q$ is homotopic to an essentially unique filtered diagonal approximation. Secondly, consider the map $S^{\infty} \times Q \stackrel{\pi_{O}}{\rightarrow} Q \stackrel{d_{0}}{\rightarrow} Q \times Q$, where $\pi_{Q}$ represents projection, and $Z_{2}$ acts only on the first component of $S^{\infty} \times Q$ and by transposition on $Q \times Q$. Then $d_{Q} \circ \pi_{Q}$ is equivariantly homotopic to an essentially unique filtered equivariant diagonal approximation.

A diagonal approximation can be used in the obvious way to define a product in the spectral sequence of $Q$. An equivariant diagonal approximation can be used to define operations in the spectral sequence. The latter construction proceeds in direct analogy with the construction of Steenrod squares for regular $C W$-complexes, replacing the cellular cochain complex of the $C W$-complex with the $E_{1}$ level of the spectral sequence of $Q$.

The category of convergent filtered spaces to be defined includes (up to filtered homotopy type) the Serre filtration of the total space of a fibration over a regular $C W$-complex, the Milgram-Dold-Lashof filtration of the classifying space of a topological monoid, and a special case of the filtered spaces used in deriving the fiber-square (Eilenberg-Moore) spectral sequence.

The content of this paper comprises part of the author's doctoral dissertation at Princeton University. 


\section{Cellularly filtered spaces}

Definition 1.1. Suppose we are given a convergent filtration of $X$ and a collection of subspaces, called cells, such that

(a) Each cell $e$ is contained in some minimal filtration $X_{n}$, and is accordingly called an $n$-cell. ( $\stackrel{e}{e}$ will denote $e_{n}-e_{n-1}$, and this will be called the interior of e.)

(b) Each $X_{n}$ is a union of cells.

(c) $X$ has the topology coherent with the cells, and each $X_{n}$ has the topology coherent with its cells.

(d) The intersection of two $n$-cells is a subset of $X_{n-1}$.

(e) For each $n$-cell $e$, there is a deformation $R_{e}: e \times I \rightarrow e$ relative to $e_{n-1}$ which represents $e_{n-1}$ as a neighborhood deformation retract (NDR) in $e$ as in definition 6.2 of [6], and with the property that for any cell $f$, $R_{e}((e \cap f) \times I) \subseteq f .\left(r_{e}: e \rightarrow e\right.$ will denote $\left.R_{e} \mid e \times 1.\right)$

(f) Each cell $e$ is acyclic as a filtered space. There is a contraction $E_{e}: e \times I \rightarrow e$ in the filtered sense (i.e. $\left.E_{e}\left(e_{n} \times I\right) \subseteq e_{n+1}\right)$ such that for any

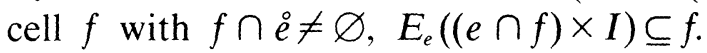

Then we will say that the filtration of $X$ is cellular (with respect to the given collection of cells), or, loosely, that $X$ is a cellularly filtered (CF) space.

Definition 1.2. Any union of cells of $X$ will be called a subcomplex.

Definition 1.3. A subcomplex $K$ is complete if for any cell $e$, $K \cap \ddot{e} \neq \varnothing$ implies that $e \subseteq K$.

Notice that if for any cell $e$, each $e_{k}$ is a subcomplex of $X$, then the second parts of (e) and (f) are automatic. This condition is the first "redundant restriction" imposed on regular $C W$-complexes in Chapter II of [1].

Notice also that for any $C F$ space $X, X_{0}$ is just a union of contractible components, each being a 0 -cell. $X$ has the topology coherent with $\left\{X_{n}\right\}$, and each $X_{n}$ is an NDR in $X_{n+1}$.

EXAmPle 1.4. Any regular $C W$-complex with the skeletal filtration and the closed $C W$ cells is a $C F$ space.

EXAmple 1.5. $X=C^{r} A$ (the iterated cone on any given space A) or $C^{\infty} A$, where $X_{n}=C^{n+1} A$.

EXAMPLE 1.6. Given any cellularly filtered space $X$ we can define a $C F$ space $X^{(k)}$ by giving $X$ a "shifted upward" filtration, i.e. $X_{n}^{(k)}=X_{n-k}$ $(n \geqq k),=X_{0}$ otherwise. Of course shifting the filtration downward by $k$ does not work, unless the components of $X_{k}$ are contractible. 
The "regularity" Condition 1.1(f) will be central in the derivation of an acyclic carrier theorem for $C F$ spaces, and so in the construction of filtered approximations to such nice maps as diagonal maps. Curiously, nonregular $C W$-complexes have filtered diagonal approximations, even when the skeletal filtrations are shifted downward, but not always when the filtration is shifted upward. As an example, let $X=X_{3}=$ the torus, $X_{1}=$ a basepoint, $X_{2}=S^{1} \vee S^{1}$. Then $(X \times X)_{3}=X \vee X$, but it is known that the diagonal map of a space is homotopic to a map into the wedge iff the space is of Lusternik-Schnirelmann reduced category $\leqq 1$.

EXAMPLE 1.7. In $\S 4$ of [7] Steenrod gives an iterative construction of the universal bundle $E_{G}$ and the classifying space $B_{G}$ of a topological monoid $G$ with base point. $D_{0}=$ a point, $E_{0}=D_{0} \times G$, and, for $n \geqq 1$, $D_{n}=$ the reduced cone on $E_{n-1}$, and $E_{n}=D_{n} \times G$ with identification of points of $E_{n-1} \times G$ and points of $E_{n-1}$ corresponding to the natural action of $G$ on $E_{n-1}$. When $G$ is discrete, $E_{G}$ becomes a cellularly filtered space if we regard each cone $D_{n} \times\{g\}$ as an $n$-cell. This does not induce a reticulation (with acyclic cells) of $B_{G}$, although in this case both $E_{G}$ and $B_{G}$ can be given the structure of simplicial complexes with skeletal filtration. When $G$ is not discrete the reticulation we have given of $E_{G}$ does not satisfy 1.1(c). Cf. 2.4 .

The following theorem and its proof are just rewordings of Lemma 4.4 of [1].

THEOREM 1.8. Let X be a cellularly filtered space. Then each compact subset $C$ of $X$ is contained in a subcomplex which is the union of a finite number of cells. Thus $X$ is convergently filtered.

Proof. We will show that $C$ meets the interiors of only finitely many cells $e^{1}, \cdots, e^{n}$. Thus $C \subseteq e^{1} \cup \cdots \cup e^{n}$. First we show that $C \subseteq$ some $X_{m}$. Suppose this is not true, and $\left\{c_{1}\right\}$ is a sequence of points of $C$ of strictly increasing filtration. Then $\left\{c_{i}\right\}$ and any subset of it meets each $X_{m}$ in a finite, thus closed, subset, so it is closed in $X . \quad\left\{c_{l}\right\}$ has a limit point $c$ in $C$, which must belong to it, but this contradicts the fact that $\left\{c_{i}\right\}-\{c\}$ is also closed.

Next we show that for each $m, C$ meets the interiors of only finitely many $m$-cells. If this were not true, there would be a sequence $\left\{c_{i}\right\}$ of points of $C$ in the interiors of distinct $m$-cells. Any subset of the form $\left\{c_{i}\right\}-\{c\}$ of $\left\{c_{i}\right\}$ would meet each cell of $X_{m}$ in $\varnothing$ or in one point, thus it would be closed in $X_{m}$. As above, this leads to a contradiction.

THEOREM 1.9. If $X$ and $X^{\prime}$ are cellularly filtered then so is $X \times X^{\prime}$, using the product cells and the "tensor product" filtration. 
Proof. Of course $X \times X^{\prime}$ is meant to denote the product in the category of compactly generated Hausdorff spaces. To prove 1.1(c), suppose $S \subset X \times X^{\prime}$ intersects each $e \times e^{\prime}$ in a closed subset. Let $C$ be a compact subset of $X \times X^{\prime}$. The projection of $C$ on $X, \pi_{X}(C)$, is a compact subset of $X$, thus it is contained in a finite union $e^{1} \cup \cdots \cup e^{n}$. Similarly, $\pi_{X^{\prime}}(C) \subseteq e^{\prime 1} \cup \cdots \cup e^{\prime m}$. Then

$$
S \cap C=S \cap\left(\pi_{X}(C) \times \pi_{X^{\prime}}(C)\right) \cap C=S \cap\left(e^{1} \times e^{\prime 1} \cup \cdots \cup e^{n} \times e^{\prime m}\right) \cap C,
$$

which is a closed subset of $C$. Since $S$ intersects each $C$ in a closed subset, $S$ is closed in $X \times X^{\prime}$. Similarly, each $\left(X \times X^{\prime}\right)_{n}$ has the topology coherent with the product cells of filtration $\leqq n$. To prove part (e), let $e$ be an $n$-cell of $X$, and $e^{\prime}$ an $m$-cell of $X^{\prime}$. Since $\left(e, e_{n-1}\right)$ and $\left(e^{\prime}, e_{m-1}^{\prime}\right)$ are NDR pairs, it is a standard result that the product $\left(e \times e^{\prime},\left(e \times e^{\prime}\right)_{m+n-1}\right)$ is an NDR pair. (Cf. for instance Theorem 6.3 of [6].) In fact, $R_{e \times e^{\prime}}$ can be chosen so that for any $\left(x, x^{\prime}\right) \in e \times e^{\prime}$, we have $R_{e \times e^{\prime}}\left(\left(x, x^{\prime}\right) \times I\right) \subseteq$ $R_{e}(x \times I) \times R_{e^{\prime}}\left(x^{\prime} \times I\right)$. Then for any cells $f$ of $X$ and $f^{\prime}$ of $X^{\prime}$,

$$
\begin{aligned}
R_{e \times e^{\prime}}\left(\left(e \times e^{\prime}\right) \cap\left(f \times f^{\prime}\right) \times I\right)= & R_{e \times e^{\prime}}\left((e \cap f) \times\left(e^{\prime} \cap f^{\prime}\right) \times I\right) \\
& \subseteq R_{e}(e \cap f \times I) \times R_{e^{\prime}}\left(e^{\prime} \cap f^{\prime} \times I\right) \\
& \subseteq f \times f^{\prime} .
\end{aligned}
$$

To prove part (f), recall the well-known result that the product of acyclic filtered spaces is acyclic, using $E_{e} \times 1$ followed by $1 \times E_{e^{\prime}}$ for $E_{e \times e^{\prime}}$. Now, suppose $\left(f \times f^{\prime}\right) \cap\left(e \stackrel{\circ}{\times} e^{\prime}\right)=\left(f \times f^{\prime}\right) \cap\left(\stackrel{\bullet}{e^{\prime} \times \dot{e}^{\prime}}\right) \neq \varnothing$. Then $f \cap \ddot{e} \neq \varnothing$ and $f^{\prime} \cap e^{\prime} \neq \varnothing$. Thus

$$
\begin{aligned}
E_{e \times e^{\prime}}\left(\left(e \times e^{\prime}\right) \cap\left(f \times f^{\prime}\right) \times I\right) & =E_{e \times e^{\prime}}\left((e \cap f) \times\left(e^{\prime} \cap f^{\prime}\right) \times I\right) \\
& \subseteq E_{e}(e \cap f \times I) \times E_{e^{\prime}}\left(e^{\prime} \cap f^{\prime} \times I\right) \subseteq f \times f^{\prime} .
\end{aligned}
$$

Notice that if $X$ and $X^{\prime}$ have the property that each stage in the filtration of any cell is a subcomplex, then this is true for $X \times X^{\prime}$ also.

DEFINITION 1.10. If $X$ and $Y$ are cellularly filtered spaces then a carrier $C$ from $X$ to $Y$ is an assignment to each cell $e$ of $X$ of a cell $C_{e}$ of $Y$, such that if $e \cap f \neq \varnothing$ then $C_{e} \cap \dot{C}_{f} \neq \varnothing . \quad C$ is filtered if for each $n$-cell $e, C_{e} \subseteq Y_{n}$. If $f: X \rightarrow Y$ we say $f$ is carried by $C$ if for each $e, f(e) \subseteq C_{e} . \quad f$ is called a cellular map if it has a filtered carrier.

Definition 1.11. Let $Z_{2}=\{1, T\}$ have the discrete topology, each point being a 0 -cell. A $Z_{2}$-action on the cells of $X$ is a representation of $Z_{2}$ by permutations of the set of cells such that $T$ of an $n$-cell is an 
$n$-cell. The action is free if for each $e, T e \neq e$. We call a choice of exactly one cell from each such pair a free basis of cells for $X$.

Definition 1.12. A $Z_{2}$-action on $X$ is cellular if it has a carrier from $Z_{2} \times X$ to $X$ which is a $Z_{2}$-action on the cells of $X$. We will call it free if this action on the cells is free. The trivial action is defined by $T x=x$ for all $x$.

If $W$ has a free $Z_{2}$-action and $X$ the trivial action, then $W \times X$ has a free $Z_{2}$-action, $Z_{2}$ acting componentwise.

The following will serve as our basic "acyclic carrier" theorem. The reference to property $(\mathrm{P})$ may be deleted if $V$ has the property that each filtration of any cell is a subcomplex.

THEOREM 1.13. Let $U$ and $V$ be cellularly filtered spaces, $K a$ subcomplex of $U, C$ a carrier from $U$ to $V, F: K \rightarrow V$ a function carried by $C$. Suppose also that $F$ has the property

(P) If $e$ is a cell of $U$, and $f$ a cell of $K$, then $F(e \cap f) \subseteq C_{e}$. Let $g$ be an $n$-cell of $U$ whose $(n-1)$-filtration is contained in $K$. Then $F$ can be extended to $g$, in such a way that $F(g) \subseteq C_{g}$ and $(\mathrm{P})$ holds when $K$ is replaced by $K \cup g$.

Proof. Recall the notations $r_{e}$ and $E_{e}$ from 1.1. $F\left(g_{n-1}\right) \subseteq C_{g}$ by (P). Since this is contractible to some point $*$ in $C_{g}$ we see we can extend $F$ to $g$. This can be done in such a way that $F[g]=*$ if $r_{g}[g] \notin g_{n-1}$, otherwise $F[g] \in E_{C_{8}}\left(F\left[r_{g}[g]\right] \times I\right)$. Now suppose $e$ is a cell of $U$ and

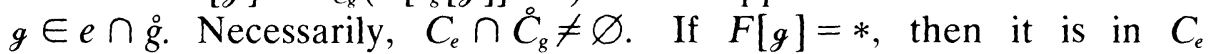
according to 1.1(f). If $F[g] \neq *$, then according to $1.1(\mathrm{e}), r_{g}[g] \in e$; by (P), $F\left[r_{g}[g]\right] \in C_{e} \cap C_{g}$; and by $1.1(\mathrm{f})$ it follows that $F[g]$, being a point of $E_{C_{R}}\left(\left[r_{g}[g]\right] \times I\right)$, is in $C_{e}$.

THEOREM 1.13'. If in $1.13 F: K \rightarrow V$ is filtered, then $F$ can be extended so as to be filtered on $\mathrm{g}$.

Proof. Repeat the argument above, using the fact that $F\left(g_{n-1}\right) \subseteq$ $\left(C_{g}\right)_{n-1}$, and the fact that $\left(C_{g}\right)_{n-1}$ is contractible to $*$ in $\left(C_{g}\right)_{n}$.

THEOREM 1.13". If in 1.13 we have a free cellular action of $Z_{2}$ on $U$ and a cellular $Z_{2}$-action on $V$, if $C$ is equivariant, and if $F$ is equivariant (and possibly filtered), then $F$ can be extended to $g \cup T g$ so as to be equivariant (and filtered).

Proof. Repeat the proof of 1.13 (or $1.13^{\prime}$ ) for $g$, then define $F$ on $T g$ by $F[T g]=T F[g]$. 
Of course such theorems can be applied in the usual way to yield the following results.

COROLlary 1.14. Let $U$ and $V$ be cellularly filtered spaces (with $Z_{2}$ acting on the cells of $V$ and freely on the cells of $U$ ). Let $C: U \rightarrow V$ be an (equivariant) carrier, $F: U \rightarrow V$ an (equivariant) function carried by $C$, and $K$ a complete subcomplex of $U$. Then $F$ is (equivariantly) homotopic to a filtered function by a homotopy carried by $C$. If $F$ is filtered on $K$, this homotopy may be chosen to be constant on $K$. If $F^{\prime}$ is another (equivariant) function carried by $C$, then $F$ and $F^{\prime}$ are (equivariantly) homotopic by a homotopy carried by $C$. If $F$ and $F^{\prime}$ are both filtered on $K$, this homotopy may be chosen to be filtered on $K \times I$. If $F$ and $F^{\prime}$ agree on $K$, this homotopy may be chosen to be constant on $K \times I$.

This corollary in turn may be applied in the usual way to yield the following two.

Corollary 1.15. Let $X$ be a cellularly filtered space. Then the diagonal map of $X$ is homotopic to a filtered map $\tilde{d}_{X}$ by a homotopy carried by the diagonal carrier. Any two such maps are homotopic by a filtered homotopy carried by this carrier. If $f: X \rightarrow Y$ is a cellular map carried by $C$ then $\tilde{d}_{Y} \circ f$ is homotopic to $(f \times f) \circ \tilde{d}_{X}$ by a filtered homotopy carried by $C \times C$.

COROLlaRy 1.16. Let $W$ be a regular $C W$-complex with free cellular action $\left(\right.$ e.g. $\left.S^{\infty}\right)$. Then the map $W \times X \stackrel{\pi_{X}}{\rightarrow} X \stackrel{d}{\rightarrow} X \times X$ is equivariantly homotopic to a filtered map $\bar{d}_{X}$ by a homotopy carried by the carrier $D_{w \times e}=e \times e$. Any other such map is homotopic to $\bar{d}_{X}$ by an equivariant filtered homotopy carried by this carrier. If $f: X \rightarrow Y$ is a cellular map carried by $C$ then $W \times X \stackrel{1 \times f}{\longrightarrow} W \times Y \stackrel{\bar{d}_{Y}}{\longrightarrow} Y \times Y$ is homotopic to $(f \times f) \circ \bar{d}_{X}$ by an equivariant filtered homotopy carried by $D C$, where $D C_{\omega \times e}=C_{e} \times C_{e}$.

2. Quasi-product spaces. The following generalizes the notion of a fiber bundle over a cellularly filtered space.

Definition 2.1. Suppose $X$ is $C F$, and to each cell $e$ of $X$ there is assigned a space $Y_{e}$. Suppose also that for each pair $e, f$ with $e \cap f \neq \varnothing$ there is given a function $\alpha_{e, f}:(e \cap f) \times Y_{e} \rightarrow Y_{f}$, such that the following hold:

(a) If $\alpha_{e, f, x}$ represents the map from $Y_{e}$ to $Y_{f}$ determined by $\alpha_{e, f, x}[y]=\alpha_{e, f}[x, y]$, then $\alpha_{f, g, x}{ }^{\circ} \alpha_{e, f, x}=\alpha_{e, g, x}$, i.e., these maps are coherent.

(b) Each $\alpha_{e, f, x}$ has a continuous right inverse $\alpha_{e, f, x}^{-1}$, and this 
assignment of right inverses can be done continuously (using the compact-open topology for function spaces) and coherently, so that $\alpha_{e, f, x}^{-1} \circ \alpha_{f, g, x}^{-1}=\alpha_{e, g, x}^{-1}$.

Let $Q^{\prime}=\Sigma e \times Y_{e}$, where $\Sigma$ denotes topological sum. Let $\sim$ denote the equivalence relation on $Q^{\prime}$ determined by the relations $(x, y) \sim$ $\left(x, \alpha_{e . f \times x}[y]\right)$. Let $Q=Q^{\prime} / \sim$, with the quotient topology, and let $q: Q^{\prime} \rightarrow Q$ be the quotient map. Then $Q$ is called the quasi-product of $X$ and the $Y_{e}$ 's determined by the $\alpha$ 's.

When $e$ and $f$ are understood, we will abbreviate $\alpha_{e, f, x}$ to $\alpha_{x}$.

Note that there is a well-defined $\operatorname{map} Q \stackrel{p}{\rightarrow} X$. We give $Q$ the filtration $Q_{n}=p^{-1}\left(X_{n}\right)$. This is a convergent filtration by NDR's.

We could have given an alternate description of a quasi-product by starting with such a $p$ which has a trivialization $e \times Y_{e} \rightarrow p^{-1}(e)$ over each cell. This leads to coherent maps $\alpha_{e, f}$ as in (a). We would also then require the "uniformity" condition (b) on the $\alpha$ 's.

It may be convenient to think of $Q$ as being decomposed into "quasi-cells", each being of the form $q\left(e \times Y_{e}\right)$. $Q$ has the topology coherent with the quasi-cells.

EXAMPLE 2.2. Let $F \rightarrow E \stackrel{p}{\rightarrow} B$ be a fibration over the regular $C W$-complex $B$. Up to fiber homotopy equivalence, we may assume that $p$ is a fiber bundle with a trivialization over each cell of $B$ and structure group $G$. Let $X=B$ and for any cell $e$ of $B$ let $Y_{e}=F$. If cell $f$ is on the boundary of cell $e$, let $\alpha_{e . f}: f \times F \rightarrow F$ be the coordinate transformation determined by the trivializations over $f$ and $e$. Each $\alpha_{e, f, x}$ will correspond to an element of $G$, and $\alpha_{e, f, x}^{-1}$ will correspond to its inverse. The quasi-product so constructed will be precisely the fiber bundle employed.

Observation 2.3. Let $G$ be an $H$-space with homotopy identity $e$ which is nondegenerate (contained in $G$ by a cofibration). Let $G^{n} \equiv G \times G \times \cdots \times G, G^{0} \equiv\{e\}$. Let $\Delta^{n}$ be the standard $n$-simplex $\equiv$ $\left\{\left(t_{1}, \cdots, t_{n}\right) \in R^{n} ; 0 \leqq t_{1} \leqq t_{2} \leqq \cdots \leqq t_{n} \leqq 1\right\}, \Delta^{0} \equiv\{0\}$. The Milgram-DoldLashof classifying space for $G$ is

$$
B M_{G} \equiv \bigcup_{n=0}^{\infty}\left(B M_{G}\right)_{n}=\left(\sum_{n=0}^{\infty} \Delta^{n} \times G^{n}\right) / \sim,
$$

where the identifications are

$$
\begin{array}{r}
\left(t_{1}, \cdots, t_{n}, g_{1}, \cdots, g_{n}\right) \sim\left(t_{1}, \cdots, \widehat{t_{t+1}}, \cdots, t_{n}, g_{1}, \cdots, g_{i-1}, g_{i} g_{i+1}, g_{i+2}, \cdots, g_{n}\right) \\
\text { if } t_{l}=t_{i+1}
\end{array}
$$




$$
\begin{aligned}
& \left(t_{1}, \cdots, t_{n-1}, 1, g_{1}, \cdots, g_{n}\right) \sim\left(t_{1}, \cdots, t_{n-1}, g_{1}, \cdots, g_{n-1}\right) \\
& \left(0, t_{2}, \cdots, t_{n}, g_{1}, \cdots, g_{n}\right) \sim\left(t_{2}, \cdots, t_{n}, g_{2}, \cdots, g_{n}\right) .
\end{aligned}
$$

We will now reconstruct $B M_{G}$ (up to filtered homotopy type) as a quasi-product.

Construction 2.4. Let $v_{0}=(0,0,0, \cdots), v_{1}=(0,1,0, \cdots), \cdots$, be the ordered vertices of $\Delta^{\infty}=U \Delta^{n}$, where $\Delta^{n}$ has vertices $v_{0}, \cdots, v_{n}$. Let $\Delta^{\prime \infty}=$ the first barycentric subdivision of $\Delta^{\infty}$. In $\left|\Delta^{\infty}\right|$, define $s \sim t$ if there is an order-preserving simplicial map $f: \Delta^{n} \rightarrow \Delta^{\infty}$ such that $|f|[s]=t$, and extend $\sim$ so that it is an equivalence relation. Let $B M_{*}=\left|\Delta^{\infty}\right| / \sim$. Let $X=\left|\Delta^{\prime x}\right| / \sim^{\prime}$, where $\sim^{\prime}$ denotes the equivalence relation on $\left|\Delta^{\prime \infty}\right|$ corresponding to $\sim$ on $\left|\Delta^{\infty}\right|$. Give $X$ the skeletal filtration. Notice that $X$ is homotopically equivalent to $B M_{*}$ as a filtered space by means of the obvious deformation, for each $n$, of $\left|\Delta^{\prime n}\right|$ to $\left|\Delta^{n}\right|$ in $\left|\Delta^{n+1}\right|$ relative to $\left|\Delta^{n}\right|$. Notice also that $X$ is contractible as a filtered space. In fact, $B M_{*}$ can be viewed as the classifying space of the trivial group using the construction given above.

Let $h$ denote the composition $\left|\Delta^{\infty}\right| \rightarrow\left|\Delta^{\prime \infty}\right| \rightarrow X$. If $e$ is an $n$-cell of $X$ whose inverse image under $h$ is contained in $\left|\left(\Delta^{\infty}\right)_{n}\right|$, let $Y_{e}=G^{n}$, otherwise let $Y_{e}=G^{n+1}$. Let $\alpha$ 's as in 2.1 be determined by the following system of identifications, using identity maps where these do not apply.

For a face $f$ of $\Delta^{n}$ containing $v_{0}$, but not $v_{t}$, let $\alpha_{\Delta^{n}, h(f)}: h(|f|) G^{n}$ $\rightarrow G^{n-1}$ be the map

$$
\alpha\left[t, g_{1}, \cdots, g_{n}\right]=\left(g_{1}, \cdots, g_{i-1}, g_{i}, g_{i+1}, g_{i+2}, \cdots, g_{n}\right),
$$

and

$$
\alpha_{t}^{-1}\left[g_{1}, \cdots, g_{1-1}, g, g_{1+2}, \cdots, g_{n}\right]=\left(g_{1}, \cdots, g_{i-1}, e, g, g_{1+2}, \cdots, g_{n}\right) \text {. }
$$

If $f$ does not contain $v_{0}$, let $\alpha\left[t, g_{1}, \cdots, g_{n}\right]=\left(g_{2}, \cdots, g_{n}\right)$ and $\alpha_{t}^{-1}\left[g_{2}, \cdots, g_{n}\right]=\left(e, g_{2}, \cdots, g_{n}\right)$. Notice that the $\alpha$ 's and $\alpha_{t}^{-1}$ 's are independent of $t$.

The quasi-product so determined will be denoted by $B_{G}$. It is homotopically equivalent to the Milgram-Dold-Lashof $B M_{G}$ as a filtered space, by means of the deformations mentioned above (using the identity on $G^{n}$ ). Notice that the projection $B_{G} \rightarrow X$ transforms via these homotopy equivalences to the map $B M_{G} \rightarrow B M_{*}$ induced by the trivial monoid homomorphism $G \rightarrow *$. Indeed, a homomorphism $G \rightarrow H$ will induce a map $B_{G} \rightarrow B_{H}$, (which will be a cellular fiber map, c.f. 2.7) which in the case $H=*$ is the quasi-product projection $q: B_{G} \rightarrow B_{*}=X$.

We now return to our discussion of quasi-products in general.

THEOREM 2.5. If $Q$ is a quasi-product over $X$ and $\bar{Q}$ a quasi-product over $\bar{X}$ then $Q \times \bar{Q}$ is a quasi-product over $X \times \bar{X}$. 
Proof. Straightforward.

In particular, allowing $\bar{Q}$ to be merely a cellularly filtered space $(\bar{X} \times a$ point $)$ we obtain

COROLlaRY 2.6. For each cellularly filtered space $M$ and quasiproduct $Q \stackrel{p}{\rightarrow} X$ there is a quasi-product $M \times Q \stackrel{1 \times p}{\longrightarrow} M \times X$, where $M \times Q$ has the "tensor product" filtration.

DEFinition 2.7. If $Q \stackrel{p}{\rightarrow} X$ and $\bar{Q} \stackrel{\bar{s}}{\rightarrow} \bar{X}$ are quasi-products then a fiber map $F: Q \rightarrow \bar{Q}$ is a map such that if $p\left[q_{1}\right]=p\left[q_{2}\right]$ then $\bar{p}\left[F\left[q_{1}\right]\right]=\bar{p}\left[F\left[q_{2}\right]\right]$. Thus $F$ induces a map $a: X \rightarrow \bar{X} . \quad F$ is called cellular if $a$ is cellular.

Now we can define carriers and $Z_{2}$-actions for quasi-product spaces. We repeat definitions $1.10-1.12$, replacing the notion of a cell $e$ by a quasi-cell $q\left(e \times Y_{e}\right)$, of $\stackrel{e}{e}$ by $q\left(\dot{e} \times Y_{e}\right)$, and of a cellular map by a cellular fiber map.

THEOREM 2.8. Suppose $Q \stackrel{q}{\rightarrow} X$ and $\bar{Q} \stackrel{\bar{q}}{\rightarrow} \bar{X}$ are quasi-products, $a: X \times I \rightarrow \bar{X}$ is carried by $C$, and $F_{0}: Q \rightarrow \bar{Q}$ is a fiber map which projects to $a_{0}$. Suppose also that $F_{0}$ factors through $q$ on each quasi-cell. That is, we have continuous functions $b_{e}: e \times Y_{e} \rightarrow Y_{C_{e}}$ such that for each pair e, $f$ with $e \cap f \neq \varnothing, \alpha_{a_{0}[x]} b_{e}[x, y]=b_{f}\left[x, \alpha_{x} y\right]$, and for each $e$ the following diagram commutes.

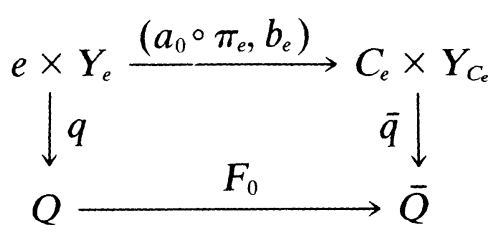

Then $F_{0}$ can be extended to a fiber map $F: Q \times I \rightarrow \bar{Q}$ which projects to a, and which factors through $q \times 1$ on each quasi-cell as follows, with $\alpha_{a[x, t]} B_{e}[x, y, t]=B_{f}\left[x, \alpha_{(x, t)} y, t\right]$ for appropriately defined $\alpha_{a[x, t]}: Y_{C_{e}} \rightarrow Y_{C_{e}}$ and $B_{e}: e \times Y_{e} \times I \rightarrow Y_{C_{e}}$.

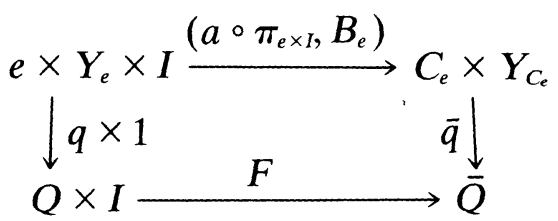


Proof. Let $H_{C_{e}}$ be the space of maps from $Y_{C_{e}}$ to $Y_{C_{e}}$, with the compact-open topology. We will construct, for each $e$, a map $G_{e}: e \times I \rightarrow H_{C_{e}}$, and define $B_{e}[x, y, t]=\left(G_{e}[x, t]\right)\left[b_{e}[x, y]\right]$. For some $n \geqq 0$, assume inductively that the $G$ 's have been defined for $e$ 's of filtration $<n$, so that $F$ is defined on $Q_{n-1} \times I$. Let $e$ be an $n$-cell of $X$. Of course $G_{e}$ must have the constant value $1_{Y_{C_{e}}}$ on $e \times 0$. We define $G_{e}$ on $e_{n-1} \times I$ as follows. For each cell $f$ of filtration $<n$ with $e \cap f \neq \varnothing$ define $G_{e}$ on $(e \cap f) \times I$ by $G_{e}[x, t]=\alpha_{a[x, t]}^{-1} G_{f}[x, t] \alpha_{a_{0}[x]}$. We must show that this leads to a definition of $F$ on $q \times 1\left((e \cap f) \times Y_{e} \times I\right)$ compatible with the way it is already defined on $q \times 1\left(f \times Y_{f} \times I\right)$. Let $(x, y) \in$ $(e \cap f) \times Y_{e}$. Then

$$
\begin{aligned}
F[q \times 1[x, y, t]] & =\bar{q}\left(a \circ \pi_{e \times I}, B_{e}\right)[x, y, t] \\
& =\bar{q}\left[a[x, t], \alpha_{a[x, t]}^{-1} G_{f}[x, t] \alpha_{a_{0}[x]} b_{e}[x, y]\right] \\
& =\bar{q}\left[a[x, t], \alpha_{a[x, t]} \alpha_{a[x, t]}^{-1} G_{f}[x, t] \alpha_{a_{0}[x]} b_{e}[x, y]\right] \\
& =\bar{q}\left[a[x, t], G_{f}[x, t] \alpha_{a_{0}[x]} b_{e}[x, y]\right] \\
& =\bar{q}\left[a[x, t], G_{f}[x, t] b_{f}\left[x, \alpha_{x} y\right]\right]=F\left[q\left[x, \alpha_{x} y, t\right]\right] .
\end{aligned}
$$

Thus we have $G_{e}$ defined on $e \times 0 \cup e_{n-1} \times I$. Since this is contractible, we can extend $G_{e}$ to $e \times I$. Thus we can define $B_{e}$ for each $n$-cell $e$, and so we can extend $F$ to $Q_{n} \times I$.

Now consider $Q \times I$ as a quasi-product. We have $F: Q \times I \rightarrow \bar{Q}$ carried by the carrier $C_{e \times e^{\prime}}^{\prime}=C_{e} . \alpha_{(x, t)}$ above should be interpreted as a coordinate transformation when cells of $Q \times I$ intersect. In general, define $\alpha_{e \times e^{\prime}, f \times f^{\prime},(x, t)}=\alpha_{e, f, x}=\alpha_{x}$. Now, for $(x, y, t) \in(e \cap f) \times Y_{e} \times I$, we have

$$
\begin{aligned}
\alpha_{a[x, t]} B_{e}[x, y, t] & =\alpha_{a[x, t]} G_{e}[x, t] b_{e}[x, y] \\
& =\alpha_{a[x, t]} \alpha_{a[x, t]}^{-1} G_{f}[x, t] \alpha_{a_{0}[x]} b_{e}[x, y] \\
& =G_{f}[x, t] \alpha_{a_{0}[x]} b_{e}[x, y] \\
& =G_{f}[x, t] b_{f}\left[x, \alpha_{x} y\right] \\
& =B_{f}\left[x, \alpha_{(x, t)} y, t\right] .
\end{aligned}
$$

Observation 2.9. In the situations with which we are primarily concerned, simpler versions of 2.8 may be used. When the $\alpha$ 's of $\bar{Q}$ are independent of $\bar{x}$, each $G_{e}$ is the constant assignment of $1_{Y_{C_{e}}}$, and the condition that $F_{0}$ factors through $q$ is unnecessary to demonstrate the existence of $F$, although $F$ may then not factor through $q \times 1$ either.

The condition that $\alpha_{a_{0}[x]} b_{e}[x, y]=b_{f}\left[x, \alpha_{x} y\right]$ is automatically satisfied under certain conditions. Since $q[x, y]=q\left[x, \alpha_{x} y\right]$, 


$$
\begin{aligned}
& F_{0}[q[x, y]]=\bar{q}\left[\left(a_{0} \circ \pi_{e}, b_{e}\right)[x, y]\right]=\bar{q}\left[a_{0}[x], b_{e}[x, y]\right] \\
& =\bar{q}\left[a_{0}[x], \alpha_{a_{0}[x]} b_{e}[x, y]\right]
\end{aligned}
$$

must be equal to

$$
F_{0}\left[q\left[x, \alpha_{x} y\right]\right]=\bar{q}\left[\left(a_{0} \circ \pi_{f}, b_{f}\right)\left[x, \alpha_{x} y\right]\right]=\bar{q}\left[a_{0}[x] \cdot b_{f}\left[x, \alpha_{x} y\right]\right] .
$$

If $a_{0}$ has the property that whenever $x \in f, a_{0}[x] \in \dot{C}_{f}$, then since $q$ is $1-1$ on $\stackrel{\circ}{f}_{f} \times Y_{C_{f}}, \alpha_{a_{0}[x]} b_{e}[x, y]=b_{f}\left[x, \alpha_{x} y\right]$ for $x \in f$, which suffices in the proof of 2.8. If the $\alpha_{x}$ 's for $\bar{Q}$ are all $1-1$, then $\bar{q}$ is $1-1$ and the equality is again demonstrated. In fact, in this case the condition that $F$ factors through $q \times 1$ is also automatic.

COROllary 2.10. For a quasi-product $Q \rightarrow X$, the diagonal map is homotopic to a filtered fiber map by a fiber homotopy carried by the diagonal carrier.

Proof. Let $a_{0}: X \rightarrow X \times X$ be the diagonal map, $F_{0}: Q \rightarrow Q \times Q$ the diagonal map. $F_{0}$ factors through $q$ as the diagonal map for each $e \times Y_{e}$. The condition $\alpha_{a_{0}[x]} b_{e}[x, y]=b_{f}\left[x, \alpha_{x} y\right]$ reduces to $\left(\alpha_{x} y, \alpha_{x} y\right)=$ $\left(\alpha_{x} y, \alpha_{x} y\right)$. Since $a_{0}$ extends to a homotopy $a: X \times I \rightarrow X \times X$ to a filtered map carried by the diagonal carrier, the result follows.

Corollary 2.11. Let $Q \rightarrow X$ be a quasi-product. Then the composition $W \times Q \stackrel{\pi_{O}}{\longrightarrow} Q \stackrel{d_{O}}{\longrightarrow} Q \times Q$ is equivariantly homotopic to a filtered fiber map by a fiber homotopy carried by the carrier $D_{\omega \times e}=e \times e$ :

Proof. This follows as above, using 1.16. When $G_{\omega \times e}$ is extended from $\omega \times e \times 0 \cup(\omega \times e)_{n-1} \times I$ to $\omega \times e \times I, G_{T \omega \times e}$ should be extended identically.

THEOREM 2.12. Suppose the hypotheses of 2.8 hold for $a: X \times I \rightarrow \bar{X}$ and $a^{\prime}: X \times I \rightarrow \bar{X}$, where $a, a^{\prime}$ are both carried by $C, a_{0}=a_{0}^{\prime}$, and $a_{1}$ and $a_{1}^{\prime}$ are filtered. Then $F_{1}$ is homotopic to $F_{1}^{\prime}$ by a filtered fiber homotopy $\bar{H}$ carried by $C$.

Proof. $F$ and $F^{\prime}$ pieced together give a homotopy $H_{0}: Q \times I \rightarrow \bar{Q}$ between $F_{1}$ and $F_{1}^{\prime}$, carried by $C$, which projects to $A_{0}: X \times I \rightarrow \bar{X}$, which is $a$ and $a^{\prime}$ pieced together. Thus $A_{00}=a_{1}$ and $A_{01}=a_{1}^{\prime}$. Notice that $H_{0}$ satisfies the hypotheses of 2.8 since it satisfies the conclusions. We can find a homotopy $A: X \times I \times I \rightarrow \bar{X}$ carried by $C$ between $A_{0}$ and a filtered map $A_{1}, A$ being constant on $X \times\{0,1\} \times I$. By 2.8 there is a fiber map $H: Q \times I \times I \rightarrow \bar{Q}$ which projects to $A$. Then $\bar{H}$ is gotten by using $H|Q \times 0 \times I, H| Q \times I \times 1$, and $H \mid Q \times 1 \times I$. 
Corollary 2.13. Any two maps constructed using 2.10 are homotopic by a filtered fiber homotopy carried by the diagonal carrier. The same holds for any two maps constructed using 2.11, the homotopy being equivariant.

Proof. For the second statement, use the variation of 2.12 in which $X \times I$ is replaced by $W \times X \times I$ and $A$ and $H$ are constructed equivariantly.

3. The fiber-square spectral sequence. In [5] Smith discusses the fiber-square (Eilenberg-Moore) spectral sequence. Let the space $B$ be fixed as the base of all projections considered. For notational simplicity we will write projections in the form $F \stackrel{F}{\rightarrow} B$, using $F$ for the total space as well as the map. One notion which Smith uses extensively is the suspension of a fibration $F$, which we will denote by $\mathscr{S F}$. The total space is the union of the ordinary suspensions of the fibers, and is not to be confused with the suspension of the total space, $S F$. Similarly, for a fiber map between fibrations $M \rightarrow N$, the mapping cone $N \cup \mathscr{C}(M)$ has as total space the union of the mapping cones of the map's restrictions to each fiber of $M$. This is distinct from the mapping cone of the map between the total spaces, $N \cup C(M)$. The fiber map sequence $M \rightarrow N \rightarrow N \cup \mathscr{C}(M)$ will be called a cofibration sequence. Finally, one may construct the mapping cylinder of a fiber map, with total space the union of the mapping cylinders of the maps between individual fibers.

In what follows we will place a bar over expressions for suspensions to denote spaces or fibrations homotopically equivalent or fiber homotopically equivalent to the spaces or fibrations represented by the unbarred expressions. For our purpose it will not be necessary to work in the category which Smith uses, $(\text { Top } / B)_{*}$.

Let

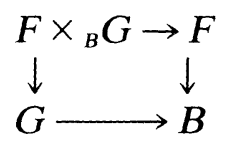

be a fiber-square, that is, $F$ is a fibration and $F \times{ }_{B} G$ is the fibered product of $F$ and $G$. Assume also that $B$ is simply-connected and $H^{*}(F), H^{*}(G)$ are of finite type as modules over $H^{*}(B)$. For any $n$ there are natural epimorphisms

$$
\left(\mathscr{S}^{n} F\right) \times{ }_{B} G \rightarrow \mathscr{S}^{n}\left(F \times{ }_{B} G\right) \rightarrow S^{n}\left(F \times{ }_{B} G\right) .
$$

For a subbundle $A$ of $\mathscr{S}^{n} F$ we will denote the image of $A \times{ }_{B} G$ in $S^{n}\left(F \times{ }_{B} G\right)$ by $A \wedge G$. This allows us to translate any filtration of $\mathscr{S}^{n} F$ by subbundles to a filtration of $S^{n}\left(F \times{ }_{B} G\right)$. 
Smith defines a display of $F$ of length $n$ as a sequence of cofibration sequences

$$
\begin{aligned}
F= & F_{0} \longrightarrow K_{0} \longrightarrow F_{-1} \\
& F_{-1} \longrightarrow K_{-1} \longrightarrow F_{-2} \\
& \vdots \\
& F_{-n+1} \rightarrow K_{-n+1} \rightarrow F_{-n}
\end{aligned}
$$

such that each $H^{*}\left(K_{-i}\right)$ is projective over $H^{*}(B)$ and each induced map $H^{*}\left(K_{-1}\right) \rightarrow H^{*}\left(F_{-1}\right)$ is onto. Displays of $F$ of any length exist. Smith points out that for $K_{-\imath}$ we may use $F_{-\imath} \times B \stackrel{\pi_{B}}{\rightarrow} B$ and for $F_{-i} \rightarrow K_{-i}$ the map $F_{-t} \stackrel{1 \times F_{-1}}{\longrightarrow} F_{-i} \times B$.

Given any cofibration sequence $M \rightarrow N \rightarrow N \cup \mathscr{C}(M)$, we have the mapping cylinder inclusion $N \cup \mathscr{C}(M) \rightarrow \overline{\mathscr{T}} M$. The pair $(\overline{\mathscr{S}} M, N \cup \mathscr{C}(M))$ thus obtained can be considered (via a fiber homotopy equivalence) as the pair in the following picture, which applies to each fiber.

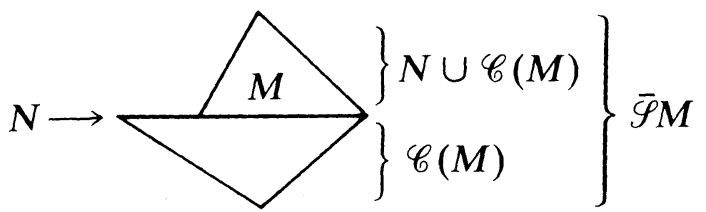

A display of $F$ thus gives rise to a filtration of $\overline{\mathscr{S}}^{n} F$

$$
F_{-n} \subseteq \overline{\mathscr{S}} F_{-n+1} \subseteq \overline{\mathscr{S}}^{2} F_{-n+2} \subseteq \cdots \subseteq \overline{\mathscr{S}}^{n-1} F_{-1} \subseteq \overline{\mathscr{S}}^{n} F_{0}
$$

where $\quad \overline{\mathscr{S}}^{r} F_{-n+r}=\overline{\mathscr{S}}^{r}\left(K_{-n+r+1} \cup \mathscr{C}\left(F_{-n+r+1}\right)\right)=\overline{\mathscr{S}}^{r} K_{-n+r+1} \cup \mathscr{C}\left(\overline{\mathscr{S}}^{r} F_{-n+r+1}\right)$, that is, $\overline{\mathscr{S}}^{r} F_{-n+r}$ is the mapping cone obtained from the $r$-fold "suspension" of the fiber map $F_{-n+r+1} \rightarrow K_{-n+r+1}$. This filtration of $\overline{\mathscr{S}}^{n} F$ results as above in a filtration of $\bar{S}^{n}\left(F \times{ }_{B} G\right)$.

$$
\begin{aligned}
F_{-n} \times{ }_{B} G \subseteq \bar{S}\left(F_{-n+1} \times{ }_{B} G\right) \subseteq \bar{S}^{2}\left(F_{-n+2} \times{ }_{B} G\right) \subseteq \cdots \subseteq \bar{S}^{n-1}\left(F_{-1} \times{ }_{B} G\right) \\
\subseteq \bar{S}^{n}\left(F_{0} \times{ }_{B} G\right)
\end{aligned}
$$

The spectral sequence of this filtered space is denoted $E(n)$. Larry Smith demonstrates that this is independent, up to isomorphism, of the display of $F$ chosen. The stable limit of the $E(n)$, that is, the spectral sequence $E_{r}^{-p q} \equiv E_{r}^{n-p q}(n), \quad n>p+1, \quad$ is the fiber-square spectral sequence, with $E_{2}^{-F q} \approx \operatorname{Tor}_{H}^{-p q_{(B)}}\left(H^{*}(F), H^{*}(G)\right)$ and $E \Rightarrow H^{*}\left(F \times{ }_{B} G\right)$.

THEOREM 3.1. Suppose $H^{*}(F)$ is of projective dimension $\nu-1$ over $H^{*}(B)$. Then displays of any length $m$ may be chosen for $F$ in which $F_{-m+k}$ and $K_{-m+k}$ are cones for $k \leqq m-\nu$. 
Proof. In Proposition 4.2 of [5] it is demonstrated that

$$
H^{*}\left(K_{-m}\right) \rightarrow H^{*}\left(\overline{\mathscr{S}} K_{-m+1}\right) \rightarrow \cdots \rightarrow H^{*}\left(\overline{\mathscr{S}}^{m} K_{0}\right) \rightarrow H^{*}\left(\overline{\mathscr{S}}^{m} F\right) \rightarrow 0
$$

is a partial projective resolution of $H^{*}\left(\overline{\mathscr{S}}^{m} F\right)$ over $H^{*}(B)$. Thus we may assume $H^{*}\left(\overline{\mathscr{S}}^{m-\nu} K_{-\nu}\right) \rightarrow H^{*}\left(\overline{\mathscr{S}}^{m-\nu+1} K_{-\nu+1}\right)$ is 0 . This map factors as

$$
\begin{aligned}
H^{*}\left(\overline{\mathscr{Y}}^{m-\nu} K_{-\nu}\right) \approx & H^{*}\left(\overline{\mathscr{S}}^{m-\nu} F_{-\nu}, \overline{\mathscr{S}}^{m-\nu-1} F_{-\nu-1}\right) \stackrel{\text { onto }}{\longrightarrow} H^{*}\left(\overline{\mathscr{S}}^{m-\nu} F_{-\nu}\right) \\
& \stackrel{1-1}{\longrightarrow} H^{*}\left(\overline{\mathscr{Y}}^{m-\nu+1} F_{-\nu+1}, \overline{\mathscr{Y}}^{m-\nu} F_{-\nu}\right) \approx H^{*}\left(\overline{\mathscr{S}}^{m-\nu+1} K_{-\nu+1}\right) .
\end{aligned}
$$

Thus $H^{*}\left(\overline{\mathscr{Y}}^{m-\nu} F_{-\nu}\right) \approx 0$, and so $H^{*}\left(F_{-\nu}\right) \approx 0$. Then we can replace $K_{-\nu}$ by $F_{-\nu}$, since $H^{*}\left(F_{-\nu}\right)$ is projective over $H^{*}(B)$ and $H^{*}\left(F_{-\nu}\right) \stackrel{\text { onto }}{\longrightarrow} H^{*}\left(F_{-\nu}\right)$. $F_{-\nu+1}$ will then be $\mathscr{C}\left(F_{-\nu}\right)$. Now proceed inductively.

THEOREM 3.2. Let $F$ be as in $3.1, n \geqq \nu$, and choose a display for $F$ as in 3.1. Then $\bar{S}^{n}\left(F \times{ }_{B} G\right)$ has a decomposition as a $C F$ space with cells $F_{-n} \wedge G$ and $\mathscr{C}\left(\overline{\mathscr{S}}^{k-1} K_{-n+k}\right) \wedge G, k \geqq 1$.

Proof. By 3.1 we can choose a display for $F$ for which $F_{-n}$ is a cone. Then $F_{-n} \wedge G$ will also be a cone. Now consider the 1-filtration of $\overline{\mathscr{S}}^{n} F, \overline{\mathscr{S}} F_{-n+1}$. It is represented in the following picture.

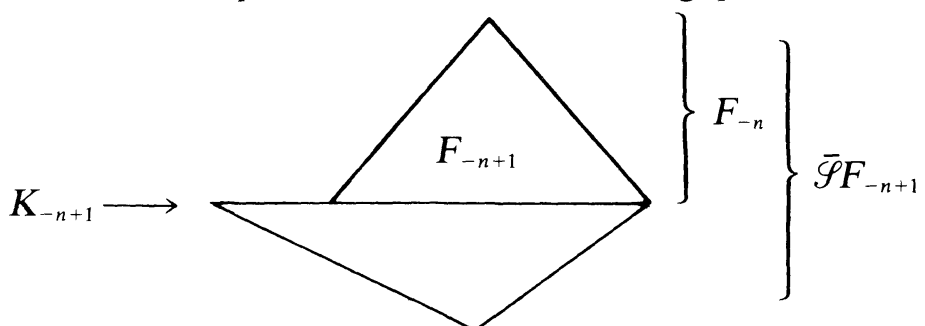

This is obtained by attaching $\mathscr{C}\left(K_{-n+1}\right) . \quad \mathscr{C}\left(K_{-n+1}\right) \wedge G$ is a cone. Obviously the 0 -filtration of $\mathscr{C}\left(K_{-n+1}\right) \wedge G$, which is $F_{-n+1} \wedge G$, is contractible in this 1-cell.

In general, for $k>1$ the $k$ 'th filtration of $\overline{\mathscr{S}}^{n} F$ is obtained from the $k-1$ st as in the following picture.

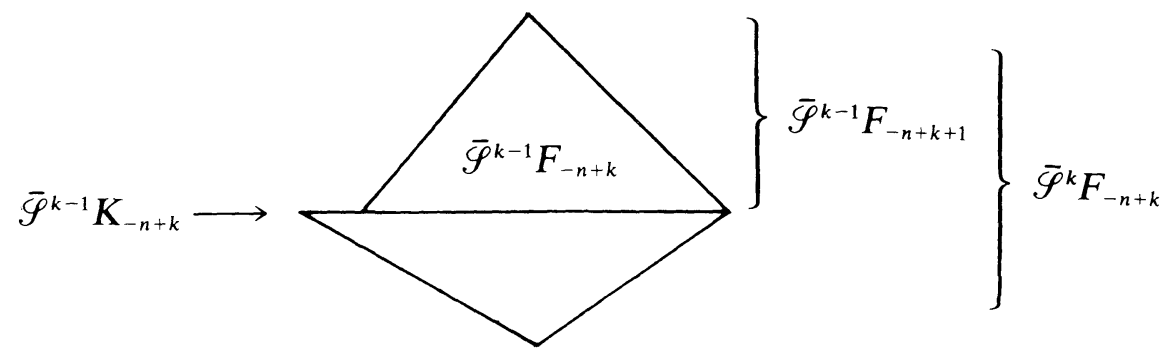


Attach the cone on $\overline{\mathscr{Y}}^{k-1} K_{-n+k}$, that is, on a sub- $k-1$-fold "suspension" of $\overline{\mathscr{S}}^{k-1} F_{-n+k-1}$. Such a sub-k-1-fold "suspension" has the property that it intersects $F_{-n}=K_{-n+1} \cup \mathscr{C}\left(F_{-n+1}\right)$ in a subcone of $\mathscr{C}\left(F_{-n+1}\right)$ and intersects each $\mathscr{C}\left(\overline{\mathscr{S}}^{\prime-1} K_{-n+j}\right), j<k$, in a subcone. Then the canonical contractions of the cones $\mathscr{C}\left(F_{-n+1}\right) \wedge G$ and $\mathscr{C}\left(\overline{\mathscr{S}}^{i-1} K_{-n+j}\right) \wedge G$ and of the base of each $\mathscr{C}\left(\overline{\mathscr{S}}^{\prime-1} K_{-n+1}\right) \wedge G$ in this cone restrict to contractions of each filtration of $\mathscr{C}\left(\overline{\mathscr{S}}^{k-1} K_{-n+k}\right) \wedge G$ in the next one. Since $\mathscr{C}\left(\overline{\mathscr{S}}^{k-1} K_{-n+k}\right) \wedge G$ is filtered by NDR's, these contractions give rise to a filtered contraction of $\mathscr{C}\left(\overline{\mathscr{S}}^{k-1} K_{-n+k}\right) \wedge G$ which, for each subbundle $K \subseteq K_{-n+k}$, keeps $\mathscr{C}\left(\overline{\mathscr{S}}^{k-1} K\right) \wedge G$ in itself.

The deformation $R_{\mathscr{G}\left(\bar{g}^{k-1} K_{-n+k) \wedge G}\right.}$ of the $k$-cell may be derived from the obvious deformation of a cone in itself which ends with the lower half being retracted to the base. This deformation keeps each subcone in itself.

We will exploit 3.2 to define a product and natural operations in the fiber-square spectral sequence for $F$ as in 3.1. Our approach will be to use $\nu$ as the canonical length for displays of $F$, so that $E_{r}^{-p q} \equiv E_{r}^{\nu-p q}(\nu)$.

Observation 3.5. When $F$ is as in $3.1, n \geqq \nu$, then $\overline{\mathscr{S}} F_{-n+1}$ is fiber homotopically equivalent to $\mathscr{S} K_{-n+1}$. Let $S=\mathscr{S} K_{-n+1} \wedge G=$ $S\left(K_{-n+1} \times{ }_{B} G\right)$, and $p$ be the top point of $S$. The standard homotopy between the diagonal map of $S$ and a map into $(S \times p) \cup(p \times S)$ induces a similar homotopy for $\overline{\mathscr{S}}_{-n+1} \wedge G$, and thus a similar homotopy for $\overline{\mathscr{S}}^{n} F \wedge G$. This homotopy is carried by the diagonal carrier. Thus it satisfies the conclusion of 1.15 .

In Theorem 6.1 of [5] and the central Theorem of [4] Smith mentions, but does not go into the details of, a multiplication in $E$. This presumably arises from a multiplication on $\operatorname{Tor}_{H *(B)}\left(H^{*}(F), H^{*}(G)\right)$ and is compatible on the $E_{\infty}$ level with the product in $H^{*}\left(F \times{ }_{B} G\right)$. This is not the product arising from the diagonal approximation above, since products in $H^{*}\left(\bar{S}^{\nu}\left(F \times{ }_{B} G\right)\right)$ are necessarily 0 .

Smith demonstrates that, given a fiber map $F \nrightarrow \bar{F}$ and a display $\bar{F}_{0} \rightarrow \bar{K}_{0} \rightarrow \bar{F}_{-1} \rightarrow \cdots \rightarrow \bar{F}_{-n}$ for $\bar{F}$, there is a display $F_{0} \rightarrow K_{0} \rightarrow$ $F_{-1} \rightarrow \cdots \rightarrow F_{-n}$ for $F$ and morphisms making

$$
\begin{aligned}
& F_{0} \rightarrow K_{0} \rightarrow F_{-1} \rightarrow \cdots \rightarrow F_{-n} \\
& \bar{F}_{0} \rightarrow \bar{K}_{0} \rightarrow \bar{F}_{-1} \rightarrow \cdots \rightarrow \bar{F}_{-n}
\end{aligned}
$$

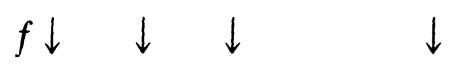

commute. Thus for $F, \bar{F}$ as in 3.1 with the projective dimension of $H^{*}(F)$ over $H^{*}(B)=\nu-1=$ the projective dimension of $H^{*}(\bar{F})$ over $H^{*}(B), f$ gives rise to a filtered map $\overline{\mathscr{S}}^{\nu} F \rightarrow \overline{\mathscr{S}}^{\nu} \bar{F}$ and a cellular map 
$\bar{S}^{\nu}\left(F \times{ }_{B} G\right) \rightarrow \bar{S}^{\nu}\left(\bar{F} \times{ }_{B} G\right)$. Of course, a cellular map is also induced by any morphism $G \rightarrow \bar{G}$.

4. Products and operations. It should be clear that the diagonal approximations and equivariant diagonal approximations for quasi-products enable us to define products and Steenrod operations in the spectral sequences. We mention a few of the details involved in the construction of the spectral operations, which parallels the construction of Steenrod squares for regular $C W$-complexes, and also give some properties of the operations.

1. The cellular cochain complex is replaced by the $E_{1}$ level of the spectral sequence: $E_{1}^{p q}=H^{p+q}\left(Q_{p}, Q_{p-1}\right)$.

2. Let $\bar{d}$ be as in the conclusion of 2.11 , and $T: S^{\infty} \rightarrow S^{\infty}$ the antipodal map. Then there are generators $\left\{w_{j} \in E_{1}^{j}{ }^{0}\left(S^{\infty}\right)=H^{\prime}\left(S^{j}, S^{j-1}\right)\right\}$ such that under the isomorphisms $E\left(S^{\infty} \times Q\right) \approx E\left(S^{\infty}\right) \otimes E(Q)$ and $E(Q \times Q) \approx E(Q) \otimes E(Q)$ we can write, for any $u \in E_{1}^{p q}(Q)$, the relation $\bar{d}^{*}[u \otimes u]=\sum_{i \geqq q}\left(1+T^{*}\right) w_{p+q-i} \otimes S^{\prime} u$. This defines operations $S^{\prime}: E_{1}^{p q} \rightarrow E_{1}^{p+i-q 2 q},(i \geqq q)$.

3. The $S^{\prime}$ pass to well-defined operations $S^{i}: E_{r} \rightarrow E_{2 r-2}, r>1$, with $S^{i} d_{r}=d_{2 r-1} S^{\prime}$, and thus to operations: $E_{\infty} \rightarrow E_{\infty}$, which are compatible with the action of the Steenrod algebra on $H^{*}(Q)$. These operations satisfy the Cartan formula and Adem relations. They agree with the operations defined by Singer in [3] in the Serre and classifying-space spectral sequences.

4. If $f: Q \rightarrow \bar{Q}$ is a cellular fiber map which factors through the projection $q$ on each quasi-cell of $Q$, then the $S^{i}$ commute with $f^{*}$ on $E_{r}$, $(r \geqq 2)$.

5. For $Q=X=a C F$ space, we can define $S_{V}^{i}: E_{1}^{p q} \rightarrow E_{1}^{p q+i}$ $(i \leqq q)$, so as to make the following diagram commute, where $X^{(q-i)}$ is $X$ with the filtration shifted upward by $q-i$ (cf. 1.6).

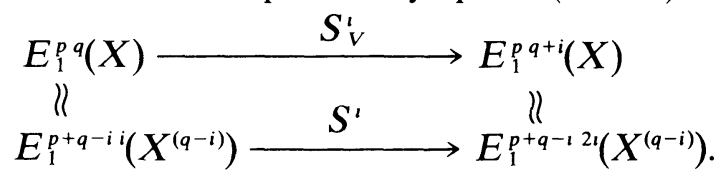

These pass to operations $S_{v}^{i}$ : $E_{r} \rightarrow E_{2 r-2}, r>1$, and $E_{\infty} \rightarrow E_{\infty}$, which are compatible with the action of the Steenrod algebra on $H^{*}(X)$. On $E_{1}^{p q}$, $S_{V}^{i}$ is $S q^{i}: H^{p+q}\left(X_{p}, X_{p-1}\right) \rightarrow H^{p+q+1}\left(X_{p}, X_{p-1}\right)$.

We end with two points about these operations in the fiber-square spectral sequence.

THEOREM 4.1. In the fiber-square spectral sequence (for $\bar{F}$ as in 3.1), on $E_{\infty}, S^{i}$ is the square for $p=0, i=q$, and is 0 otherwise.

Proof. $E^{n-p q}(n)$ is associated to a filtration of $H^{n-p+q}\left(S^{n}\left(F \times{ }_{B} G\right)\right)$, and $E^{-p q}$ is associated to the corresponding filtration of $H^{-p+q}\left(F \times{ }_{B} G\right)$. 
Since $S q^{i}$ is 0 on either of these cohomology modules for $i>q$, and is the square for $p=0, i=q$, the theorem follows.

Thus when the fiber-square spectral sequence collapses, $S^{i}$ is described by 4.1 , replacing $\infty$ by any $r \geqq 2$.

EXAMPLE 4.2. The operations $S_{V}^{i}$ in the fiber-square spectral sequence are the ones considered by Larry Smith in [5], p. 50, and Rector in [2], since they behave on $E_{1}$ as above. They are not 0 in general. An example of a fibration $F \rightarrow B$ with $H^{*}(F)$ of finite projective dimension over $H^{*}(B)$ is gotten as follows. Consider the universal bundle over $B_{G}$, where $G$ is a Lie group whose cohomology over $Z_{2}$ is the algebra on a simple system of transgressive generators, $\Delta\left(x_{i_{1}}, \cdots, x_{i_{k}}\right)$. Then $H^{*}\left(B_{G}\right)=$ $Z_{2}\left[y_{i_{1}+1}, \cdots, y_{i_{k}+1}\right]$ and the graded algebra

$$
Z_{2}\left[y_{i_{1}+1}, \cdots, y_{i_{k}+1}\right] \otimes E\left[z_{i_{1}}, \cdots, z_{i_{k}}\right]
$$

( $E$ denoting an exterior algebra) is a projective resolution of $Z_{2}$ over $H^{*}\left(B_{G}\right)$, each $y$ being in filtration 0 and each $z$ in filtration -1 . Thus $Z_{2}$ has projective dimension $k$ over $H^{*}\left(B_{G}\right)$. Suppose, as is often the case, that $S q^{i} x_{i}=x_{i_{j}+i}$. The fiber-square spectral sequence for the diagram

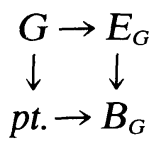

will collapse, with $\operatorname{Tor}_{H^{*}\left(B_{G}\right)}\left(Z_{2}, Z_{2}\right)=E\left[z_{i_{1}}, \cdots, z_{i_{k}}\right]=E_{\infty}, z_{i}$, being the coset of $x_{i}$.in $F^{-1} H^{i,+1} / F^{0} H^{i_{i}+1}$. Thus $S_{V}^{i} z_{i}$, must be $z_{i,+1}$.

\section{REFERENCES}

1. George E. Cooke and Ross L. Finney, Homology of cell complexes, Princeton Univ. Press, Princeton, N.J., 1967.

2. David L. Rector, Steenrod operations in the Eilenberg-Moore spectral sequence, Comm. Math. Helv., 45 (1970), 540-552.

3. William M. Singer, Steenrod squares in spectral sequences, I, II, unpublished notes, Dept. of Mathematics, Boston College, Chestnut Hill, Mass.

4. Larry Smith, On the construction of the Eilenberg-Moore spectral sequence, Bull. Amer. Math. Soc., 75 (1969), 873-878.

5. L_ Lectures on the Eilenberg-Moore spectral sequence, Lecture Notes in Mathematics no. 134. Springer-Verlag, New York, 1970.

6. Norman E. Steenrod, A convenient category of topological spaces, Michigan J. Math., 14 (1967), 133-152.

7. _ Milgram's classifying space of a topological group, Topology, 7 (1968), 349-368.

Received July 12, 1975.

UNIVERSITY OF MISSOURI - ST. LOUIS 



\section{PACIFIC JOURNAL OF MATHEMATICS}

\section{EDITORS}

RICHARD ARENS (Managing Editor)

University of California

Los Angeles, California 90024

\section{J. Dugundu}

Department of Mathematics University of Southern California Los Angeles, California 90007

D. Gilbarg and J. Milgram Stanford University Stanford, California 94305

\section{ASSOCIATE EDITORS}
E. F. BECKENBACH
B. H. NeumanN
F. WOLF
K. YoSHIDA

\section{SUPPORTING INSTITUTIONS}

UNIVERSITY OF BRITISH COLUMBIA CALIFORNIA INSTITUTE OF TECHNOLOGY

UNIVERSITY OF CALIFORNIA

MONTANA STATE UNIVERSITY

UNIVERSITY OF NEVADA

NEW MEXICO STATE UNIVERSITY

OREGON STATE UNIVERSITY

UNIVERSITY OF OREGON

OSAKA UNIVERSITY

\author{
UNIVERSITY OF SOUTHERN CALIFORNIA \\ STANFORD UNIVERSITY \\ UNIVERSITY OF HAWAII \\ UNIVERSITY OF TOKYO \\ UNIVERSITY OF UTAH \\ WASHINGTON STATE UNIVERSITY \\ UNIVERSITY OF WASHINGTON \\ AMERICAN MATHEMATICAL SOCIETY
}

The Supporting Institutions listed above contribute to the cost of publication of this Journal, but they are not owners or publishers and have no responsibility for its contents or policies.

Mathematical papers intended for publication in the Pacific Journal of Mathematics should be in typed form or offset-reproduced (not dittoed), double spaced with large margins. Underline Greek letters in red, German in green, and script in blue. The first paragraph or two must be capable of being used separately as a synopsis of the entire paper. Items of the bibliography should not be cited there unless absolutely necessary, in which case they must be identified by author and Journal, rather than by item number. Manuscripts, in duplicate, may be sent to any one of the four editors. Please classify according to the scheme of Math. Reviews, Index to Vol. 39. All other communications should be addressed to the managing editor, or Elaine Barth, University of California, Los Angeles, California, 90024.

100 reprints are provided free for each article, only if page charges have been substantially paid. Additional copies may be obtained at cost in multiples of 50 .

The Pacific Journal of Mathematics is issued monthly as of January 1966. Regular subscription rate: $\$ 72.00$ a year (6 Vols., 12 issues). Special rate: $\$ 36.00$ a year to individual members of supporting institutions.

Subscriptions, orders for back numbers, and changes of address should be sent to Pacific Journal of Mathematics, 103 Highland Boulevard, Berkeley, California, 94708.

PUBLISHED BY PACIFIC JOURNAL OF MATHEMATICS, A NON-PROFIT CORPORATION

Printed at Jerusalem Academic Press, POB 2390, Jerusalem, Israel.

$$
\begin{gathered}
\text { Copyright } 1976 \text { Pacific Journal of Mathematics } \\
\text { All Rights Reserved }
\end{gathered}
$$




\section{Pacific Journal of Mathematics}

Vol. 62, No. 2

February, 1976

Allan Russell Adler and Catarina Isabel Kiefe, Pseudofinite fields, procyclic

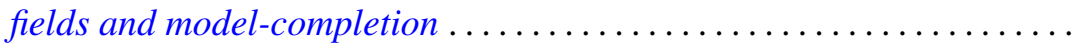

Christopher Allday, The stratification of compact connected Lie group

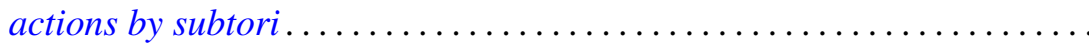

Martin Bartelt, Commutants of multipliers and translation operators .......

Herbert Stanley Bear, Jr., Ordered Gleason parts ..................

James Robert Boone, On irreducible spaces. II .....................

James Robert Boone, On the cardinality relationships between discrete

collections and open covers ............................

L. S. Dube, On finite Hankel transformation of generalized functions .......

Michael Freedman, Uniqueness theorems for taut submanifolds . . . . . . . . .

Shmuel Friedland and Raphael Loewy, Subspaces of symmetric matrices

containing matrices with a multiple first eigenvalue .............

Theodore William Gamelin, Uniform algebras spanned by Hartogs

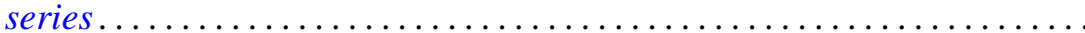

James Guyker, On partial isometries with no isometric part ............

Shigeru Hasegawa and Ryōtarō Satō, A general ratio ergodic theorem for

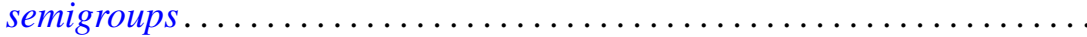

Nigel Kalton and G. V. Wood, Homomorphisms of group algebras with norm less than $\sqrt{2}$.

Thomas Laffey, On the structure of algebraic algebras...

Will Y. K. Lee, On a correctness class of the Bessel type differential operator $S_{\mu}$

Robert D. Little, Complex vector fields and divisible Chern classes ....

Kenneth Louden, Maximal quotient rings of ring extensions . .

Dieter Lutz, Scalar spectral operators, ordered $l^{\rho}$-direct sums, and the

counterexample of Kakutani-McCarthy . .

Ralph Tyrrell Rockafellar and Roger Jean-Baptiste Robert Wets, Stochastic

convex programming: singular multipliers and extended duality

singular multipliers and duality.

Edward Barry Saff and Richard Steven Varga, Geometric overconvergence of rational functions in unbounded domains ..........

Joel Linn Schiff, Isomorphisms between harmonic and P-harmonic Hardy

spaces on Riemann surfaces.

Virinda Mohan Sehgal and S. P. Singh, On a fixed point theorem of

Krasnoselskii for locally convex spaces.

Lewis Shilane, Filtered spaces admitting spectral sequence operations

Michel Smith, Generating large indecomposable continua . 